\title{
A pregnant woman with COVID-19 gives birth to premature triplets: A case report and literature review
}

\author{
ZAILI FENG ${ }^{1,2}$ \\ ${ }^{1}$ Department of Neonatology, Kunming Medical University (Dehong People's Hospital), Kunming, Yunnan 650500; \\ ${ }^{2}$ Department of Neonatology, Ruili Chinese Medicine and Dai Medical Hospital, Ruili, Yunnan 678600, P.R. China
}

Received October 20, 2021; Accepted February 11, 2022

DOI: $10.3892 / \mathrm{mi} .2022 .31$

\begin{abstract}
To the best of our knowledge, no case report has been published to date concerning the novel coronavirus disease 2019 (COVID-19) treatment plan for pregnant women with triplets, as well as on the possibility of a vertical transmission to triplets delivered prematurely by a pregnant woman with COVID-19. The present study thus describes such as case. A 29-year-old local resident, a woman with COVID-19, who was pregnant with triplets, had not moved from her residence for the past 6 months. The main symptoms mentioned by the patient on the day of admission were the following: The patient was in the 28th week of pregnancy, 2019 novel coronavirus (2019-nCoV) nucleic acid positive test $13 \mathrm{~h}$ prior to admission. Additionally, the patient had a high fever and dyspnea after admission. The patient was treated with convalescent plasma from patients with COVID-19 and methylprednisolone, at the same time her anemia, anxiety and insomnia were treated. Subsequently, the patient's condition improved, and normal body temperature was restored. Cesarean section was performed due to severe intrahepatic cholestasis of pregnancy (ICP), and due to the fetuses being located in three chorionic and amniotic cavities, and due to the fact that each of the three infants was in a different position (cephalic, breech and transverse position). Following delivery, total bile acid values and liver function improved gradually, and the 2019-nCoV nucleic acid test was negative consecutively for two tests, and the woman was then discharged
\end{abstract}

Correspondence to: Professor Zaili Feng, Department of Neonatology, Kunming Medical University (Dehong People's Hospital), 1168 Chunrong West Road, Yuhua Street, Chenggong, Kunming, Yunnan 650500, P.R. China

E-mail: fengzaili@sina.com; 1007363616@qq.com; fengzaili@qq.com

Abbreviations: ALT, alanine transaminase; AST, aspartate aminotransferase; COVID-19, novel coronavirus disease 2019; CRP, C-reactive protein; Glu, glucose (blood sugar); Hb, hemoglobin; ICP, intrahepatic cholestasis of pregnancy; IL-6, interleukin-6; NICU, a neonatal intensive care unit; PCT, procalcitonin; TBA, total biliary acid; 2019-nCoV, 2019 novel coronavirus

Key words: COVID-19, convalescent plasma from patients with COVID-19, infection, premature infants, mother of triplets, 2019-nCoV from the hospital. Each premature infant born by caesarean section was individually transported to a neonatal intensive care unit for isolation treatment, with the neonatal escorts and transport teams wearing secondary protective clothing. All three premature infants were transitioned gradually from parenteral intravenous nutrition to full oral feeding. 2019-nCoV nucleic acid test results were negative for all infants. 2019-nCoV IgM test results were negative and $\mathrm{IgG}$ were positive for all infants. The pregnant woman and the three premature infants were successfully treated and discharged after they felt no discomfort and were negative consecutively for two 2019-nCoV nucleic acid tests. The three premature infants were monitored for growth and development until December, 2021, and eye, lung, heart, brain, liver, kidney, intestine and other organ functions were normal. The present study reports on the treatment and delivery outcomes of a woman with COVID-19, who was pregnant with triplets. The present case report demonstrated that although the pregnant woman with triplets was suffering from COVID-19, all three premature infants delivered by cesarean section did not present with intrauterine vertical infection, and abnormal growth and development were not observed. The convalescent plasma of patients with COVID-19 and methylprednisolone were effective for the treatment of a continuously high fever, and the timely treatment of complications is helpful for the treatment of patients with COVID-19.

\section{Introduction}

The novel coronavirus disease 2019 (COVID-19) is elicited by the 2019 novel coronavirus (2019-nCoV), More than 388 million individuals have been infected worldwide, posing a serious threat to human health (1). Pregnant women infected with 2019-nCoV have been reported to be at an increased risk of developing severe disease and having adverse outcomes and fetuses can also be severely affected (2). As the gestational age increases, the shape of a pregnant woman's uterus changes, lung capacity decreases, hormone levels change and immune functions are suppressed; thus, pregnant women who are infected with COVID-19 are at a high risk of developing severe illness (3). During the period in which epidemic prevention and lockdown measures were implemented, the author had the opportunity to treat a pregnant woman with triplets who was also infected with 2019-nCoV; her condition gradually developed from asymptomatic infection to severe disease. 
Following treatment with convalescent plasma from patients with COVID-19, methylprednisolone, etc., all parameters measured were found to return to normal values. By 32 weeks and 5 days of pregnancy, the patient delivered by cesarean section due to intrahepatic cholestasis of pregnancy (ICP), and the three premature infants were not infected with 2019-nCoV. This case is reported herein and in addition, a review of the relevant literature is also presented.

\section{Case report}

\section{General information}

History of present illness. The patient was 29 years old and was admitted to the Ruili Chinese Medicine and Dai Medical Hospital (Ruili, Yunnan, China) due to her 2019-nCoV nucleic acid positive test $13 \mathrm{~h}$ prior to admission. The patient was G1P0 and at 28 weeks of gestation and pregnant with triplets, which were conceived naturally; regular evaluations during the course of pregnancy yielded normal results. Within 2 weeks prior to admission, the patient occasionally felt fatigued and suffered from palpitations, no fever, cough, dyspnea, chest tightness, abdominal pain or diarrhea, had a normal sense of taste and smell, a normal spirit and normal sleep patterns. The present study was approved by the Medical Ethics Committee of the Dehong People's Hospital Affiliated to the Kunming Medical University (Dehongzhou People's Hospital; approval no. DHZYYLL2021-019), Kunming, China, and informed consent was obtained from the parents of the infants and from the pregnant woman.

Past history. The patient was previously healthy, had not been vaccinated against 2019-nCoV, and no 2019-nCoV infection had been reported in her family. Repeated 2019-nCoV nucleic acid tests were negative for 6 months prior to admission.

Physical examination upon admission. The patient's physical measurements were as follows: Height, $157 \mathrm{~cm}$; weight, $59 \mathrm{~kg}$; body temperature, $36.8^{\circ} \mathrm{C}$; pulse rate, 102 beats $/ \mathrm{min}$; respiration rate, 22 beats $/ \mathrm{min}$; blood pressure, $123 / 85 \mathrm{mmHg}$; and oxygen saturation (without oxygen inhalation), 98\%. The patient's general conditions were normal, without any detected heart, lung or abdomen abnormalities during the physical examination. Additionally, occasional uterine contractions were observed upon an obstetric examination. and triplet fetal heart auscultation was normal. Furthermore, no vaginal bleeding or fluids were detected.

Examination of the patient. Routine blood and biochemical testing, as well as coagulation function testing were performed using a Roche Cobas c701 fully automatic biochemical analyzer (Roche Diagnostics), Mindray BC-6800 auto hematology analyzer (Shenzhen Mindray Bio-Medical Electronics Co., Ltd.) and a Sysmex CS5100 Coagulation Analyzer (Sysmex Corporation), respectively. All corresponding test results are presented in Tables I-III. An ABI 7500 real-time fluorescent quantitative PCR instrument (Thermo Fisher Scientific, Inc.) was used for 2019-nCoV RNA load determination. Zhijiang Biotechnology Co., Ltd. was used for the nucleic acid test, and a Ct value $<43$ was considered as a positive result; the $2019-\mathrm{nCoV}$ nucleic acid detection kit (fluorescent PCR method, National Machinery Note: 20203400064) of Shengxiang Biotechnology Co., Ltd. was used for nucleic acid testing, and a $\mathrm{Ct}$ value $<40$

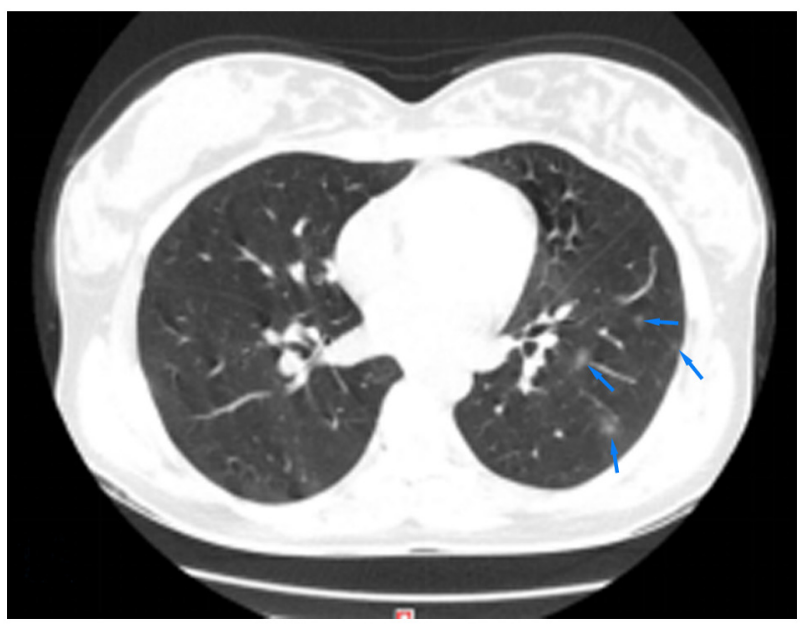

Figure 1. Computed tomography report of the female patient pregnant with triplets on the 4th day of the disease course: The thorax is symmetrical. There are repeated small sections of ground glass shadows in the upper, lower and right middle lobes of the lungs, with some consolidation, thickened blood vessels and blurred edges, consistent with common COVID-19 symptoms.

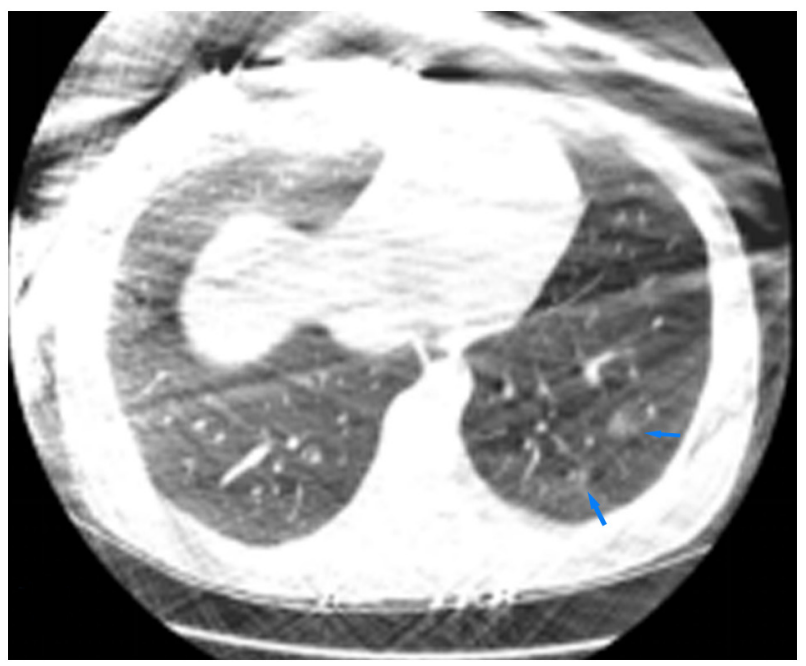

Figure 2. Computed tomography report on the 4th day of the disease course: Several lesions were covered by protective lead clothing, affecting the observation. The thorax is symmetrical. There are repeated small sections of ground glass shadows in the upper, lower and right middle lobes of the lungs, with some consolidation, thickened blood vessels and blurred edges, consistent with commonly reported COVID-19 symptoms.

was considered as a positive result; each sample was analyzed with both reagent tests, and the final report was the one with the highest positive result. Subsequently, a GE 16-slice spiral CT OPTIMA CT520Pro scanner (Cytiva) was used for the acquisition of patient chest computed tomography (CT) scans. The patient was required to lie in the supine position for the acquisition of the CT scans. The corresponding scan parameters were as follows: Collimator, $0.625 \mathrm{~mm}$; tube voltage, $120 \mathrm{kv}$; tube current, 139 mAs; Fov, 38 cm; W 1,400 Hu; L-480 $\mathrm{Hu}$. The CT images of the lungs are demonstrated in Fig. 1. According to the CT report on the 4th day of the disease course (Figs. 1 and 2), some lesions were covered by protective lead clothing, affecting the observation. The thorax was symmetrical. There were repeatedly small sections of ground glass 

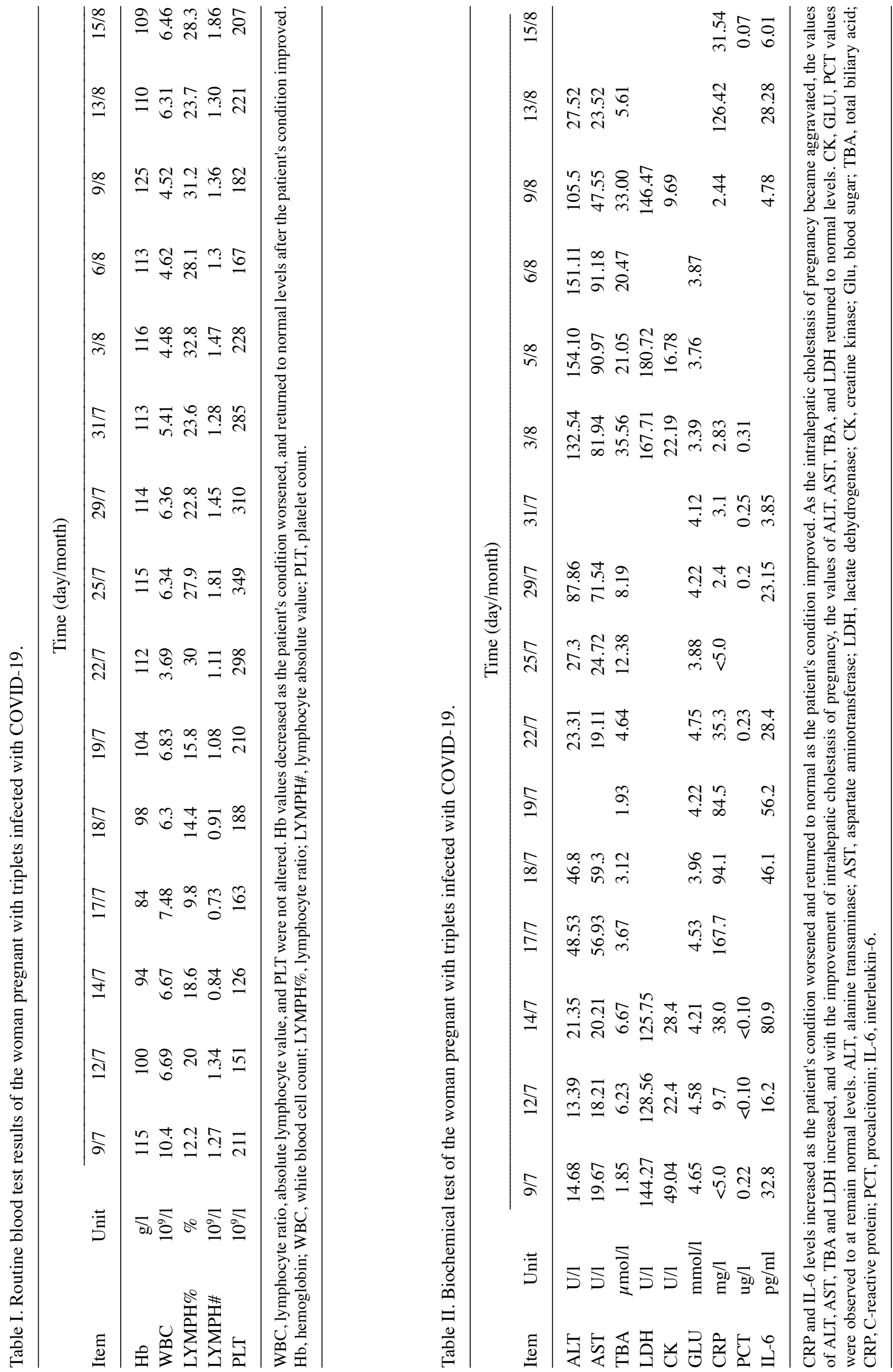


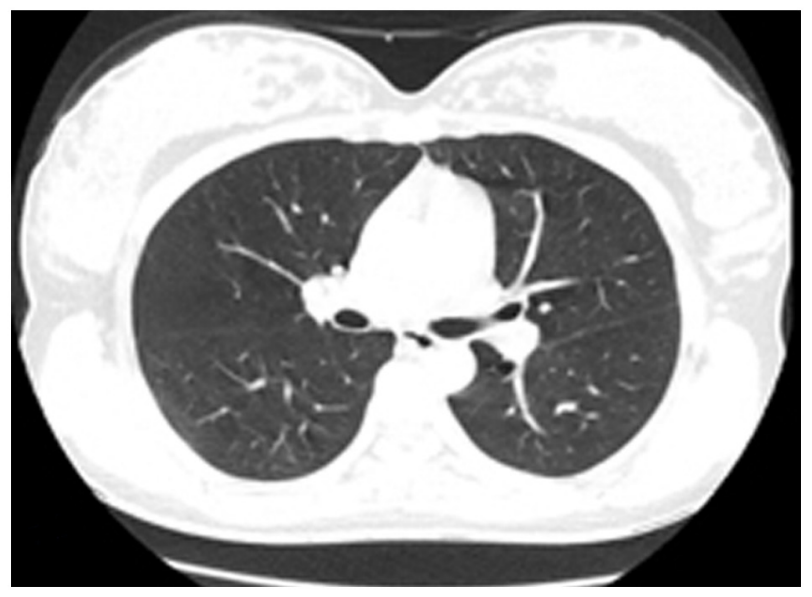

Figure 3. Computed tomography image on the 52nd day of the disease course following treatment: The lung lesions have been absorbed and have been notably improved.

shadows in the upper, lower and right middle lobes of the lungs, with some consolidation, thickened blood vessels and blurred edges, being consistent with common COVID-19-related effects. CT imaging was also performed on the 52nd day of the disease course following treatment (Fig. 3); at this time, the lung lesions were absorbed and the conditions were significantly improved in comparison with the initial clinical observations. During hospitalization, the ultrasound examination of the patient revealed pulmonary consolidation, abnormal pleural line and pulmonary edema. The ultrasound reports of the premature infants were normal. All three fetuses did not experience intrauterine distress after repeated fetal heart monitoring.

Diagnosis and treatment of the patient. The joint diagnosis and treatment medical team members were the following: The Chinese Epidemic Prevention and Control Command Expert Group, Respiratory Science Group, Obstetrics Group, Neonatology group and Critical Medicine Science Group. After consultation with these expert groups, diagnosis and treatment were conducted based on the patient's medical history, epidemiological investigation, and changes in symptoms and signs. The diagnosis, classification and main treatments administered are presented in Table IV. Respiratory tract isolation, contact isolation and daily fetal heart monitoring were performed until the infant delivery was completed. The patient began to develop skin itching, increased transaminase alanine transaminase (ALT) and aspartate aminotransferase (AST) levels, and increased bile acid levels at 31 weeks and 4 days of pregnancy. Following the exclusion of other liver diseases, the patient was diagnosed with severe ICP. Subsequently, analysis by the medical staff of the joint diagnosis and treatment team revealed that anti-COVID antibodies could be detected, and the nucleic acid $\mathrm{Ct}$ values were close to negative, indicating that the patient underwent the recovery period. The patient uterine height was measured at $38 \mathrm{~cm}$ and the abdominal circumference was $104 \mathrm{~cm}$. Patient cardiopulmonary compression was also clearly detectable. The gestational age was 32 weeks and 5 days. Premature infant delivery was expected without any lethal incidents. Due to pregnancy and 
Table IV. Diagnosis, classification and main patient treatments.

\section{Date}

(day/month/year)

Conditions

Diagnosis

Asymptomatic infection with COVID-19 and palpitations.

Vital signs were stable.

$11 / 07 / 2021$

Mild sore throat, cough

COVID-19 and expectoration.

Vital signs

were stable.

$12 / 07 / 2021$

$16 / 07 / 2021$

$22 / 07 / 2021$

Dry cough, no fatigue, no palpitations, no dyspnea, no fever.

COVID-19

(ordinary type)

COVID-19

(ordinary type)

Severe intrahepatic

cholestasis of pregnancy

29/08/2021
Itchy skin, vital signs were stable.

No discomfort, vital signs were stable. Discharge.
COVID-19

(ordinary type)

COVID-19

(severe type)
Magnesium sulfate suppresses uterine contractions and protects cranial nerves of fetuses $(11 / 07 / 2021$ to 13/07/2021).

Reduced-molecular-weigh heparint sodium prevents microvascular sodium thrombosis $(11 / 07 / 2021$ to 16/07/2021).

Intermittent nasal catheter low flow oxygen inhalation (12/07/2021 to $15 / 07 / 2021)$.

Methylprednisolone sodium succinate (15/07/2021 to $18 / 07 / 2021)$ Infusion with COVID-19 convalescen plasma (1,800 $\mathrm{ml}$ of type A virus) (13/07/2021 to 17/07/2021).

High-flow oxygen inhalation through the nose, restricting activities (16/07/2021 to 21/078/2021). Pumping morphine $(0.5 \mathrm{mg} / \mathrm{h})$ at night to improve patient's sleep (16/07/2021 to 21/07/2021).

Infusion A (positive) RBC suspension $3 \mathrm{U}$ to improve anemia (16/07/2021 to $18 / 07 / 2021)$.

Actively improve the preparations for cesarean section and pre-start the follow-up treatment of premature (16/97/2021)

Nasal cannula oxygen inhalation (22/07/2021 to 27/07/2021) Low molecular weight heparin sodium prevents microvascular thrombosis

$(27 / 07 / 2021$ to $03 / 08 / 2021)$

Ursodeoxycholic acid tablets (04/08/2021 to 11/08/2021) Cesarean section (11/08/2021).

Post-cesarean section nursing $(11 / 08 / 2021$ to 29/08/2021).

Nucleic acid test results were consecutively negative (22/08/2021, 23/08/2021, 28/08/2021, 29/08/2021).

Examination of lung computed tomography scan before discharge indicated that the lesions were absorbed (29/08/2021).

Completion of discharge procedures and leaving the hospital (29/08/2021).
COVID-19 infection, and as each of the three infants was in a different position (cephalic, breech and transverse position); it was decided for the pregnancy to be concluded by performing a cesarean section. Cesarean section was performed in the negative pressure ward at 32 weeks and 5 days of pregnancy, under combined spinal-epidural anesthesia. The infants were born in a single transfer single room with isolated treatment. Following a 24-h time period, with a negative nucleic acid test (three times), the newborns were transferred to the same nursing unit for treatment until discharge.

Delivery and treatment of premature infants. Three transfer teams were engaged in total, and each transfer team was responsible for the transfer of one premature infant. The preterm infants were transported using T-combination resuscitator ventilation. The premature infants were born by caesarean section. Each 
premature infant was separately transported to a neonatal intensive care unit (NICU) for isolation treatment by a neonatal escort delivery team wearing secondary protective clothing. At the time of the laparotomy, the amniotic fluid of three amniotic cavities was obtained for 2019-nCoV nucleic acid test and the results were negative. The 2019-nCoV nucleic acid test of the stool, urine, gastric juice, nasal swab and throat swab samples were completed three times at $0.5,12$ and $24 \mathrm{~h}$ following delivery. All three premature infants were negative for infection. The three premature infants were transferred to the same nursing unit for treatment until discharge. During hospitalization, 2019-nCoV nucleic acid testing was performed repeatedly and all corresponding results were negative. The blood antibody test results for all three premature infants 2019-nCoV IgM test results were negative and IgG were positive many times after birth.

Treatment results and follow-up. Following delivery, bile acid and liver functions gradually improved, the ICP was cured, and the nucleic acid tests were negative (two times). The patient was then transferred to the isolation point for 14 days for 2019-nCoV treatment, and was not found positive at the end of this time period; the patient was thus discharged. Monitoring continued until December, 2021, and after that time point, the patient was continually found negative for 2019-nCoV infection. All three premature infants were discharged after no signs of discomfort were detected and were negative consecutively for two 2019-nCoV nucleic acid tests. Repeated nucleic acid tests were negative, indicating that the three premature infants were not infected with 2019-nCoV. The three premature infants were monitored for growth and development continuously until December, 2021. Eye, lung, heart, brain, liver, kidney, intestine and other organ functions were also not found to be abnormal.

\section{Discussion}

2019-nCoV testing and monitoring. In the present case report, combined with clinical manifestations and imaging results, the diagnosis of COVID-19 was confirmed. The three premature infants reported in the present case report who were monitored until discharge, 2019-nCoV nucleic acid test results were negative and 2019-nCoV IgM test results were negative, but IgG results were positive. Nucleic acid and antibody levels of the patient and of the three infants required continuous monitoring.

It took only 28 days for the COVID-19 pathogen to be identified in China (4). Quantitative polymerase chain reaction (qPCR) is commonly used to detect 2019-nCoV, and clinical gene sequencing is commonly used for pathogen metagenomic sequencing (mNGS). Young patients, patients with asymptomatic or mild clinical symptoms are more likely to be subsequently re-infected with $2019-\mathrm{nCoV}$, whereas this is rare in patients with severe and critical COVID-19 (5). The majority of patients re-infected with 2019-nCoV have no disease progression, and the majority of patients do not present with obvious lung symptoms, thus requiring no special treatment. Isolation and observation are recommended, and the 2019-nCoV nucleic acid is required to be tested regularly (6). By analyzing the causes of 2019-nCoV re-infection, a main cause has been reported to be 2019-nCoV residual re-infection (7), or alternatively 2019-nCoV could be mainly concentrated in the lower respiratory tract, and false negatives may occur when nasopharyngeal swabs are collected (8). Multiple site sampling and repeated screening for 2019-nCoV have been reported to increase the positive diagnosis rate (9). Therefore, strict self-isolation and long-term follow-up are crucial after acquiring a negative result for 2019-nCoV (10). In the present case report, the $2019-\mathrm{nCoV}$ nucleic acid analysis results were negative following patient treatment, and no re-infection occurred. In addition, the test results for 2019-nCoV infection of the premature infants were negative.

2019-nCoV infection may cause adverse effects in pregnant women, fetuses and newborns, thus continuous monitoring of the fetus after birth has been reported to be crucial (11). In addition to 2019-nCoV nucleic acid and antibody levels, the most commonly used method for disease monitoring is low-dose chest CT scan. Previous research has reported that that a low-dose chest CT scan repeated for up to 10 times during pregnancy will not cause fetal malformations (12), and a low-dose chest CT in patients with COVID-19 plays a crucial role in screening, diagnosis, triage and follow-up (13). The characteristics of the pulmonary ultrasound imaging of COVID-19 are lung consolidation, pulmonary edema, pleural effusion and abnormal pleural line, amongst others. Lung ultrasound has been reported to be of definite value in the diagnosis of pneumonia and to be suitable for its application in lung disease screening in adults, children and newborns $(14,15)$. In the case presented herein, a low-dose spiral CT scan was used on the patient and a lung ultrasound examination was also performed. The results of the lung ultrasound examination were compared and evaluated along with those of previous lung ultrasound examinations, reducing the risk of radiation injury.

No vertical intrauterine infection of three premature infants born by cesarean section. The receptor of 2019-nCoV has been clearly researched and it is the angiotensin converting enzyme 2 (ACE2). It mainly exists in the respiratory and digestive tract. It is also widely distributed in the lymph nodes, thymus, spleen, bone marrow, liver, skin, brain and other organs. It cannot be fully excluded that the infection of the organs with ACE2 receptors may cause damage to corresponding organs (16). The respiratory tract and contact transmission route have been confirmed to be the main routes of transmission, while the fecal-oral transmission route and the vertical transmission route from-mother-to-child remain controversial. A previous study demonstrated that 2019-nCoV was detected in the saliva of patients, and part of it was active, suggesting that 2019-nCoV may still be transmitted directly or indirectly through saliva (17). Infection by touching the surface of contaminated objects is termed contact infection (18). The infection of cases in different regions of China suggests the existence of fecal-oral transmission (19).

There are several previously published studies in favor of the vertical transmission of 2019-nCoV from mother to child not being possible. One study reported that 10 infants delivered by pregnant women infected with 2019-nCoV were not infected with 2019-nCoV (20). Another study reported nine cases of 2019-nCoV-infected pregnant women. However, 2019-nCoV nucleic acid testing for amniotic fluid, fetal umbilical cord blood, neonatal nasopharyngeal swabs and breast milk yielded negative results (21). Furthermore, in a 
study on 55 pregnant women with COVID-19, no evidence of the vertical transmission of $2019-\mathrm{nCoV}$ was found in 46 newborns (22). In another study, a total of 71 pregnant women infected with 2019-nCoV were examined, and none of the newborns were found to be infected with 2019-nCoV (23). In addition, a study on 42 pregnant women with COVID-19 in the third trimester revealed that newborns were not infected with 2019-nCoV (24). In another study, 2019-nCoV RT-PCR tests were performed on 30 samples of cord blood, 26 samples of breast milk, 23 samples of amniotic fluid during childbirth and 12 samples of placenta submitted for examination, and the results were all negative (25). The author analyzed that the possible reasons for the absence of vertical transmission may be the following: i) Prevention, isolation and protection measures were taken for the pregnant women and children before and during delivery; ii) treatment of pregnant women before and during delivery minimized the pregnant women's viral load; iii) timely treatment before delivery may cause the mother to produce antibodies, and IgG antibody may exert a protective effect on the fetus through the placenta.

On the contrary, other previously published studies have suggested that 2019-nCoV transmission may occur vertically from mother to child. In a previous study on which 33 cases of neonates delivered by pregnant women with COVID-19 were examined, three cases of neonates positively tested for SARSCoV-2 in the upper respiratory tract and anus were confirmed to be infected with SARS-CoV-2; however, due to strict compliance with infection control and preventive measures during childbirth, vertical mother-to-child transmission was not fully excluded (26). Another study reported a case of a pregnant woman with COVID-19. In that study, the 2019-nCoV antibody IgG levels stated were: $140.32 \mathrm{AU} / \mathrm{ml}$, IgM: $45.83 \mathrm{AU} / \mathrm{ml}, 2 \mathrm{~h}$ after the birth of the newborn delivered by cesarean section, due to strict compliance with infection control and preventive measures during childbirth, being in support of the possibility of intrauterine vertical infection (27). The possible causes of vertical transmission in those cases of viral transmission are analyzed as follows: i) The treatment was not provided timely before delivery, and the pregnant women's viral load was too high; ii) regardless of the treatment being timely before delivery or not, the treatment was not effective, and the pregnant women's viral load was too high; iii) the immune function of the pregnant women was reduced since the SARS-CoV-2 does not cause immunity or induces weak immunity; and iv) prevention, isolation and protection measures were not taken during delivery. Based on the aformentioned studies, it can be suggested that the mother-to-child vertical transmission of 2019-nCoV may occur; however, the probability of transmission is minimal.

Body stimulation by pathogens has been reported to induce the production of large amounts of IgG. It has been demonstrated that $\operatorname{IgG}$ is able to traverse through the placenta; thus, IgG can be detected after birth, and $\operatorname{IgG}$ continues to exist for a long period of time. However, IgM, having a greater molecular weight, is not able to traverse through the placenta, and the IgM half-life is reduced. Therefore, newborns positive for IgM may indicate the possibility of vertical transmission in the uterus (28). Some researchers have suggested that even if a mother suffers from COVID-19, it is not impossible to rule out the possibility of other viral infections; thus, it is also considered that only IgM positivity is not sufficient evidence of 2019-nCoV congenital infection (27-29). The three premature infants described in the present case report were negative for 2019-nCoV nucleic acid monitoring, and 2019-nCoV IgM test results were negative, but the IgG results were positive. It was thus considered that vertical intrauterine infection did not occur. Additionally, it has not yet been determined throughout the literature, to the best of our knowledge, whether vaginal delivery or cesarean delivery is the safest method. A previous study on 42 pregnant women suffering from COVID-19 in the third trimester of pregnancy reported that $52.4 \%$ of pregnant women gave birth vaginally, and the newborns were not infected with $2019-\mathrm{nCoV}$ (24), suggesting that vaginal delivery may not increase the risk of vertical transmission. It is uncertain whether breastfeeding leads to the transmission of 2019-nCoV. A study on 48 breast milk samples revealed that one sample was positive $2019-\mathrm{nCoV}$, and none of the other samples were positive for 2019-nCoV (30). The three premature infants reported in the present study were fed with formula milk after birth, and no $2019-n \mathrm{CoV}$ infection was found. Following discharge, the mother's nucleic acid test results were negative, and breast milk test results were negative repeatedly. Breastfeeding was introduced, and formula milk was added when the breast milk was insufficient.

Convalescent plasma from patients with COVID-19 combined with methylprednisolone are effective for COVID-19 treatment and persistent high fever, and timely treatment for complications are helpful for the treatment of COVID-19. Several therapeutics have been suggested for the treatment of 2019-nCoV infection and COVID-19; however, their effectiveness and safety, particularly the safety of medication during pregnancy, remain to be further investigated $(19,31,32)$. According to the literature, the mortality rate of COVID-19 may be range between 3.1-7.2\%; however, the range in mortality rate in cases of severe COVID-19 infection is between $15.7-25.5 \%$, and in critical cases, it has been reported to be as high as $39.0-61.5 \%$ (33). In addition to timely symptomatic treatment, respiratory and circulatory support treatment, severe and critical COVID-19 patients can also use the COVID-19 patient's convalescent plasma treatment. Previous research has reported that treatment with convalescent plasma from patients with COVID-19 may reduce mortality (34). It is suggested that patients with severe and critical COVID-19 infection, particularly patients with rapid progression, be treated with convalescent plasma from patients with COVID-19 as soon as possible. A study suggested that the majority of patients with COVID-19 were treated most effectively with convalescent plasma from patients with COVID-19, following the onset of symptoms for 6-14 days, particularly after the onset of 3-5 days (35). Previous studies have revealed that the use of convalescent plasma from patients with COVID-19 may increase survival rates for critically ill patients with rapid disease progression and a continuously high viral load following antiviral and hormonal therapy, including pregnant women. Convalescent plasma may inhibit viral replication, early inflammatory response and organ tissue damage. The use of convalescent plasma from patients with COVID-19 has been recommended at an earlier stage instead of using it solely as remedial treatment $(36,37)$. 
The effects of convalescent plasma from patients with COVID-19 in the treatment of passive immunity include viral neutralization and immune regulation. The suggested mechanisms of action are, according to Hung et al (38), as follows: i) The most important component of convalescent plasma is the $\gamma$-globulin molecule, which has been reported to clear the viral load and relieve viremia; ii) the neutralizing antibody and non-neutralizing antibody of convalescent plasma may enhance the adaptive immune response mediated by T-lymphocytes; iii) the important complement regulatory proteins, including CD55 contained in the COVID-19 patient's convalescent plasma may inhibit the activity of complement $\mathrm{C} 3$ and $\mathrm{C} 5$, accelerate their decomposition, and reduce inflammatory damage to host cells. The general principle of the COVID-19 patient's convalescent plasma treatment infusion is a high antibody titer and repeated administration, being favorable for viral neutralization, without any accompanying side-effects according to a previously published study (39). In China, it is recommended that the infusion dose of convalescent plasma from patients with COVID-19 is usually $200-500 \mathrm{ml}$ (4-5 ml/ $\mathrm{kg}$ ); if the COVID-19 patient's convalescent plasma antibody titer is relatively low, an increased dose will be necessary (40). The adverse reactions from treatment with convalescent plasma of COVID-19 patients are mild, with an incidence of $12.9 \%$, and an incidence of $3.1 \%$ has been attributed to transfusion (41). Although treatment with convalescent plasma from patients with COVID-19 theoretically poses a risk of infection with 2019-nCoV, there are currently no reported cases of infection due to the use of such plasma, at least to the best of our knowledge.

Methylprednisolone inhibits the release of cytokines and inflammatory mediators, and exerts anti-inflammatory effects, and methylprednisolone acts on the hypothalamic body temperature regulation center and the heat-producing organs, resulting in the restoration of body temperature to normal levels $(42,43)$. From the above, it is known that methylprednisolone exerts anti-inflammatory and fever-limiting effects. However, it may inhibit the immune function of patients, delay virus clearance and also cause sodium retention, body fluid retention, osteoporosis and others $(42,43)$. Therefore, the use of corticosteroids is not recommended for the routine and long-term treatment of COVID-19. Methylprednisolone has been commonly used in China for a short-term period (3-5 days) and reduced-dose (1-2 mg/kg/day) treatment, particularly for patients with dyspnea and severe hypoxemia. The possible causes of anemia in patients with COVID-19 may involve bone marrow hematopoietic function possibly being inhibited to reduce red blood cell (RBC) production, or the activation of mononuclear macrophages to produce extravascular hemolysis leads to increased RBC destruction, and anemia may thus occur. Breathing difficulties become more intense, and severe cases may lead to breathing failure, lung damage, all of which can aggravate COVID-19. Additionally, in patients with a well-functioning immune system, the 2019-nCoV viral load has been reported to be more easily cleared, with the quality of sleep directly affecting immune efficiency further (44-46). Sudden COVID-19 disease has been reported to affect the psychological, physical and social functions of patients, and may lead to a gradual increase in the levels of cortisol and norepinephrine. The increase in the levels of these hormones may affect the patient's disposition, resulting in increased anxiety, affecting sleep and disease prognosis. It has also been reported that a functioning immune system is important for viral load reduction and for the promotion of a timely patient recovery, with the quality of sleep being a crucial factor affecting immunity. The immunity of patients with sleep disorders has been demonstrated to be severely damaged, leading to the weakening of the body's resistance and viral load elimination efficacy $(47,48)$.

In the present case report, the patient developed persistent high fever, fatigue, and heart palpitations. Combined with the findings of CT imaging, it was suggested that the patient's clinical condition was aggravated. The patient was then treated with convalescent plasma from patients with COVID-19; however, the patient's clinical condition did not improve. Methylprednisolone was also added to the treatment combination, and subsequently, her body temperature returned to normal levels, following 6 days of treatment. It was thus suggested that convalescent plasma from patients with COVID-19 combined with methylprednisolone was effective in treating COVID-19 and the persistent high fever. During the course of the illness, the patient was anxious and suffered from insomnia; the effect of psychotherapy was not obvious. Following morphine treatment and a moderate exercise program, the ability to sleep was improved. During the course of the disease, blood transfusion was used to correct anemia treatment, and the patient changed from severe to normal COVID-19. It is suggested that timely treatment with complications, including anxiety, insomnia, and anemia are favorable for COVID-19 treatment.

Patient with COVID-19, pregnant with triplets exhibits increased $C$-reactive protein $(C R P)$, increased interleukin (IL)-6, and decreased hemoglobin $(\mathrm{Hb})$ levels with the worsening of the clinical conditions. The majority of patients with COVID-19 have normal white blood cell counts, increased CRP levels, and a decreased number of lymphocytes. Lung CT scans demonstrate typical punctate and ground glass changes near the pleura, and some patients have liver damage. CRP is often used for the evaluation of infectious diseases. It is a sensitive indicator of inflammation, and the majority of patients with COVID-19 present with increased CRP levels. In patients with severe COVID-19 infection, levels of $\geq 10 \mathrm{mg} / \mathrm{l}$ account for $81.5 \%$ (49,50). Patients with severe COVID-19 infection often also present with increased levels of inflammatory factors (31). The early increase in the levels of D-dimer in patients with COVID-19 may be related to the inflammatory response. A sharp and sudden increase in D-dimer levels may indicate an acute inflammatory response storm, indicating that the disease may be exacerbated. The average increasement rate during COVID-19 infection has been reported to be $46.4 \%$ (51). In severe COVID-19 cases of pregnant woman, the hematopoietic function of bone marrow may be inhibited in order to reduce $\mathrm{RBC}$ production, or the activation of mononuclear macrophages. As a result, extravascular hemolysis may occur, possibly leading to increased RBC destruction and anemia.

In addition to its coagulation biological effects, platelets are also a main innate immunity component. In some patients with COVID-19, increased levels of liver enzymes and myocardial enzymes have been reported, and in several patients with critical COVID-19 infection, troponin levels may also increase $(33,52)$. In another study, it was revealed that 
the blood urea and blood creatinine levels of non-surviving patients before death increased rapidly compared with those of survivors (51). It has also been reported that the average glucose/blood sugar (Glu) levels of patients with COVID-19 are $7.4 \mathrm{mmol} / \mathrm{l}$, and $52 \%$ of patients present with increased Glu levels (52). Additionally, it has been reported that the majority of patients with COVID-19 exhibit normal serum procalcitonin (PCT) levels when admitted to hospital, and an increase in secondary bacterial infections $(33,52)$. Therefore, it is recommended that patients with COVID-19 be monitored for Glu, CRP and PCT levels, biochemical indicators, coagulation function, arterial blood gas analysis, chest imaging, cytokines.

In the present case report, the condition of the woman pregnant with triplets and infected with COVID-19 worsened gradually; the CRP and IL-6 levels increased, and the $\mathrm{Hb}$ levels notably decreased. Increased CRP, increased IL-6 and decreased $\mathrm{Hb}$ levels are more sensitive markers than the white blood cell count; the absolute value of lymphocytes, Hb, PLT and blood coagulation may indicate the aggravation of the disease. The white blood cell count, absolute value of lymphocytes, $\mathrm{Hb}$, platelet count and coagulation function were not markedly altered during the course of the disease and treatment. This may be attributed to the timely treatment when the conditions worsened.

Pregnant women with COVID-19 are more likely to suffer from ICP, leading to liver damage (53), which may be directly caused by the virus or by the systemic inflammatory response induced by the virus (33). The occurrence and exacerbation of the ICP may be related to estrogen, thyroid hormones, environment, genetics, immune factors. ICP may have adverse effects on pregnant women and fetuses.

In the present case report, the patient presented with increased total biliary acid (TBA), abnormal liver function and myocardial enzymes, and skin itching at 31 weeks and 4 days of gestation, and was diagnosed with severe ICP. Subsequent treatment with ursodeoxycholic acid tablets was not effective. The cesarean section was performed at 32 weeks and 5 days of gestation following the completion of glucocorticoid-induced lung maturation. The purpose was to decrease or avoid the toxic effects of high bile acids. Following delivery, TBA decreased, liver function improved, and ICP was cured. It was suggested that the ICP of the woman with COVID-19 who was pregnant with triplets may progress rapidly; the therapeutic effect of ursodeoxycholic acid tablets was ineffective, and childbirth was an effective treatment method.

In brief, it has been previously stated COVID-19 should not be diagnosed from clinical manifestations alone. For individuals with an epidemiological history and obvious clinical manifestations, if a nucleic acid test is negative, multiple tests need to be repeated to eliminate the possibility of false negatives. Effective sampling can ensure that the samples have a sufficient viral load. After sampling, the 2019-nCoV nucleic acid test is performed in due time, combined with the main clinical symptoms such as fever or cough for diagnosis, so false negatives are reduced (54). Additionally, COVID-19 may cause a normal flora imbalance in the patient's respiratory tract, which can be combined with multiple viral infections. Among these combined viral infections, there are more blood-borne viruses, and blood indicators monitoring needs to be intensified $(55,56)$.
In conclusion, in the present study, although the pregnant woman with triplets was infected with COVID-19, the three premature infants delivered by cesarean section did not present with intrauterine vertical infection, and abnormal growth and development were not observed. Treatment with convalescent plasma from patients with COVID-19 combined with methylprednisolone was effective for the treatment of COVID-19 and the persistent high fever, and the timely treatment of complications including anxiety and insomnia were helpful for the treatment of COVID-19. Increased CRP, increased IL-6, and decreased $\mathrm{Hb}$ levels possibly indicated that the patient's condition worsened. In addition, increased CRP, increased IL-6, and decreased $\mathrm{Hb}$ levels are more sensitive indicators of disease severity than routine blood white blood cell counts, lymphocyte ratio and absolute lymphocyte value, the $\mathrm{Hb}$ and PLT levels, and coagulation function. The ICP of the woman pregnant with triplets infected with COVID-19 progressed rapidly, and the use of ursodeoxycholic acid tablets was not an effective treatment for ICP; however, delivery proved to be safe and effective.

The present case report described the disease changes, treatment methods and treatment effects of a female patient with COVID-19 bearing triplets. In all three premature infants delivered by cesarean section, vertical intrauterine transmission did not occur. In comparison with other similar case reports, the present study has certain limitations: The characteristics of an individual case only were presented. Therefore, previously the published literature was reviewed, aiming to strengthen the understanding of the majority of medical workers on the treatment of pregnant women with triplets, infected with COVID-19 and also in relation to the outcomes of preterm infants.

The diagnosis and treatment experience and related literature review hereby reported may provide future reference for medical practitioners. The present study suggested that pregnant women with multiple babies should be treated promptly if they are suffering from COVID-19, and treatments including convalescent plasma from patients with COVID-19 and methylprednisolone, are effective for those who are suffering from COVID-19. It is also strongly suggested that the 2019-nCoV nucleic acid level, CRP, IL-6 and Hb levels should be monitored in patients with COVID-19. Additionally, the delivery of triplets from a pregnant woman infected with COVID-19 does not necessarily lead to the transmission of 2019-nCoV infection to the infants or induce infant growth and development issues. Thus, COVID-19 infection is not necessarily associated with pregnancy loss.

\section{Acknowledgements}

Not applicable.

\section{Funding}

The present study was supported by the Education Department of Yunnan Province, China (grant no. 2019J1316).

\section{Availability of data and materials}

The datasets used and/or analyzed during the current study are available from the corresponding author on reasonable request. 


\section{Author's contributions}

ZF independently completed the following work: Conception and design of the study; Acquisition, analysis, and interpretation of data; drafting the manuscript and revising it critically for content; final approval of the manuscript to be published; agreement to be accountable for all aspects of this study in ensuring that questions related to the accuracy or integrity of any part of this manuscript are appropriately investigated and resolved. The author has read and approved the final manuscript. ZF confirms the authenticity of all the raw data.

\section{Ethics approval and consent to participate}

The present study was approved by the Medical Ethics Committee of the Dehong People's Hospital Affiliated to the Kunming Medical University (Dehongzhou People's Hospital; approval no. DHZY YLL2021-019), Kunming, China, and informed consent was obtained from the parents of the children and pregnant women.

\section{Patient consent for publication}

The present case report obtained the informed consent of the patient, and the report/publishing was carried out with her authorization.

\section{Competing interests}

The author declares that there are no competing interests.

\section{References}

1. Zhu N, Zhang D, Wang W, Li X, Yang B, Song J, Zhao X, Huang B Shi W, Lu R, et al: A novel coronavirus from patients with pneumonia in China, 2019. N Engl J Med 382: 727-733, 2020.

2. Perlman S: Another decade, another coronavirus. N Engl J Med 382: 760-762, 2020.

3. Martinez-Portilla R, Smith ER, He S, Torres J, Espino S, Solis-Paredes JM and Poon LC: Young pregnant women are also at an increased risk of mortality and severe illness due to COVID-19: Analysis of the Mexican national surveillance program. Am J Obstet Gynecol 224: 404-407, 2020.

4. Cheng VCC, Wong SC, To KKW, Ho PL and Yuen KY: Preparedness and proactive infection control measures against the emerging novel coronavirus in China. J Hosp Infect 104 254-255, 2020

5. An J, Liao X, Xiao T, Qian S, Yuan J, Ye H, Qi F, Shen C, Wang L, Liu Y, et al: Clinical characteristics of recovered COVID-19 patients with re-detectable positive RNA test. Ann Transl Med 8: 1084, 2020

6. Atzrodt CL, Maknojia I, McCarthy RDP, Oldfield TM, Po J, Ta KTL, Stepp HE and Clements TP: A guide to COVID-19: A global pandemic caused by the novel coronavirus SARS-CoV-2. FEBS J 287: 3633-3650, 2020.

7. Zhang B, Liu S, Dong Y, Zhang L, Zhong Q, Zou Y and Zhang S: Positive rectal swabs in young patients recovered from coronavirus disease 2019 (COVID-19). J Infect 81: e49-e52, 2020.

8. Yao XH, He ZC, Li TY, Zhang HR, Wang Y, Mou H, Guo Q Yu SC, Ding Y, Liu X, et al: Pathological evidence for residual SARS-CoV-2 in pulmonary tissues of a ready-for-discharge patient. Cell Res 30: 541-543, 2020.

9. Deng W, Guang TW, Yang M, Li JR, Jiang DP, Li CY and Wang DX: Positive results for patients with COVID-19 discharged form hospital in Chongqing, China. BMC Infect Dis 20: 429, 2020

10. Fu W, Chen Q and Wang T: Letter to the editor: Three cases of redetectable positive SARS-CoV-2 RNA in recovered COVID-19 patients with antibodies. J Med Virol 92: 2298-2301, 2020.
11. Dang D, Wang L, Zhang C, Li Z and Wu H: Potential effects of SARS-CoV-2 infection during pregnancy on fetuses and newborns are worthy of attention. J Obstet Gynaecol Res 46: 1951-1957, 2020

12. Williams PM and Fletcher S: Health effects of prenatal radiation exposure. Am Fam Physician 82: 488-493, 2010.

13. Chung M, Bernheim A, Mei X, Zhang N, Huang M, Zeng X, Cui J, Xu W, Yang Y, Fayad ZA, et al: CT imaging features of 2019 novel coronavirus (2019-nCoV). Radiology 295: 202-207, 2020.

14. Liu J: Lung ultrasonography for the diagnosis of neonatal lung disease. J Matern Fetal Neonatal Med 27: 856-861, 2014.

15. Kurepa D, Zaghloul N, Watkins L and Liu J: Neonatal lung ultrasound exam guidelines. J Perinatol 38: 11-22, 2018.

16. Zhou P, Yang XL, Wang XG, Hu B, Zhang L, Zhang W, Si HR, Zhu Y, Li B, Huang CL, et al: A pneumonia outbreak associated with a new coronavirus of probable bat origin. Nature 579: 270-273, 2020

17. To KK, Tsang OT, Yip CC, Chan KH, Wu TC, Chan JM, Leung WS, Chik TS, Choi CY, Kandamby DH, et al: Consistent detection of 2019 novel coronavirus in Saliva. Clin Infect Dis 71: 841-843, 2020.

18. de Graaf M, Beck R, Caccio SM, Duim B, Fraaij P, Le Guyader FS, Lecuit M, Le Pendu J, de Wit E and Schultsz C: Sustained fecal-oral human-to-human transmission following a zoonotic event. Curr Opin Virol 22: 1-6, 2017.

19. Holshue ML, DeBolt C, Lindquist S, Lofy KH, Wiesman J, Bruce H, Spitters C, Ericson K, Wilkerson S, Tural A, et al: First case of 2019 novel coronavirus in the United States. N Engl J Med 382: 929-936, 2020.

20. Zhu H, Wang L, Fang C, Peng S, Zhang L, Chang G, Xia S and Zhou W: Clinical analysis of 10 neonates born to mothers with 2019-nCoV pneumonia. Transl Pediatr 9: 51-60, 2020.

21. Chen H, Guo J, Wang C, Luo F, Yu X, Zhang W, Li J, Zhao D, $\mathrm{Xu} \mathrm{D}$, Gong Q, et al: Clinical characteristics and intrauterine vertical transmission potential of COVID-19 infection in nine pregnant women: A retrospective review of medical records. Lancet 395: 809-815, 2020.

22. Dashraath P, Wong JLJ, Lim MXK, Lim LM, Li S, Biswas A, Choolani M, Mattar C and Su LL: Coronavirus disease 2019 (COVID-19) pandemic and pregnancy. Am J Obstet Gynecol 222: 521-531, 2020.

23. Prabhu M, Cagino K, Matthews KC, Friedlander RL, Glynn SM, Kubiak JM, Yang YJ, Zhao Z, Baergen RN, DiPace JI, et al: Pregnancy and postpartum outcomes in a universally tested population for SARS-CoV-2 in New York City: A prospective cohort study. BJOG 127: 1548-1556, 2020.

24. Marín Gabriel MA, Cuadrado I, Álvarez Fernández B, González Carrasco E, Alonso Díaz C, Llana Martín I, Sánchez L, Olivas C, de Las Heras S and Criado E; Neo-COVID-19 Research Group: Multicentre Spanish study found no incidences of viral transmission in infants born to mothers with COVID-19. Acta Paediatr 109: 2302-2308, 2020.

25. Elshafeey F, Magdi R, Hindi N, Elshebiny M, Farrag N, Mahdy S, Sabbour M, Gebril S, Nasser M, Kamel M, et al: A systematic scoping review of COVID-19 during pregnancy and childbirth. Int J Gynaecol Obstet 150: 47-52, 2020.

26. Zeng L, Xia S, Yuan W, Yan K, Xiao F, Shao J and Zhou W: Neonatal early-onset infection with SARS-CoV-2 in 33 neonates born to mothers with COVID-19 in Wuhan, China. JAMA Pediatr 174: 722-725, 2020

27. Dong L, Tian J, He S, Zhu C, Wang J, Liu C and Yang J: Possible vertical transmission of SARS-CoV-2 from an infected mother to her newborn. JAMA 323: 1846-1848, 2020

28. Zeng H, Xu C, Fan J, Tang Y, Deng Q, Zhang W and Long X: Antibodies in infants born to mothers with COVID-19 pneumonia. JAMA 323: 1848-1849, 2020.

29. Kimberlin DW and Stagno S: Can SARS-CoV-2 infection be acquired in utero? More definitive evidence is needed. JAMA 323: 1788-1789, 2020

30. Lackey KA, Pace RM, Williams JE, Bode L, Donovan SM, Järvinen KM, Seppo AE, Raiten DJ, Meehan CL, McGuire MA and McGuire MK: SARS-CoV-2 and human milk: What is the evidence? Matern Child Nutr 16: e13032, 2020.

31. Huang C, Wang Y, Li X, Ren L, Zhao J, Hu Y, Zhang L, Fan G, Xu J, Gu X, et al: Clinical features of patients infected with 2019 novel coronavirus in Wuhan, China. Lancet 395: 497-506, 2020.

32. Chan JF, Yuan S, Kok KH, To KK, Chu H, Yang J, Xing F, Liu J, Yip CC, Poon RW, et al: A familial cluster of pneumonia associated with the 2019 novel coronavirus indicating person-to-person transmission: A study of a family cluster. Lancet 395: 514-523, 2020. 
33. Yang X, Yu Y, Xu J, Shu H, Xia J, Liu H, Wu Y, Zhang L, Yu Z, Fang M, et al: Clinical course and outcomes of critically ill patients with SARS-CoV-2 pneumonia in Wuhan, China: A single-centered, retrospective, observational study. Lancet Respir Med 8: 475-481, 2020.

34. Hung IF, To KK, Lee CK, Lee KL, Chan K, Yan WW, Liu R, Watt CL, Chan WM, Lai KY, et al: Convalescent plasma treatment reduced mortality in patients with severe pandemic influenza A (H1N1) 2009 virus infection. Clin Infect Dis 52: 447-456, 2011.

35. ToK K, Tsang OT, Leung WS, Tam AR, Wu TC, Lung DC, Yip CC, Cai JP, Chan JM, Chik TS, et al: Temporal profiles of viral load in posterior oropharyngeal saliva samples and serum antibody responses during infection by SARS-CoV-2: An observational cohort study. Lancet Infect Dis 20: 565-574, 2020.

36. Shen C, Wang Z, Zhao F, Yang Y, Li J, Yuan J, Wang F, Li D, Yang M, Xing L, et al: Treatment of 5 critically ill patients with COVID-19 with convalescent plasma. JAMA 323: 1582-1589, 2020.

37. Pan Y, Zhang D, Yang P, Poon LLM and Wang Q: Viral load of SARS-CoV-2 in clinical samples. Lancet Infect Dis 20: 411-412, 2020 .

38. Hung IFN, To KKW, Lee CK, Lee KL, Yan WW, Chan K, Chan WM, Ngai CW, Law KI, Chow FL, et al: Hyperimmune IV immunoglobulin treatment: A multicenter double-blind randomized controlled trial for patients with severe 2009 influenza $\mathrm{A}(\mathrm{H} 1 \mathrm{N1})$ infection. Chest 144: 464-473, 2013.

39. Gutfraind A and Meyers LA: Evaluating large-scale blood transfusion therapy for the current Ebola epidemic in Liberia. J Infect Dis 211: 1262-1267, 2015

40. Joyner MJ, Wright RS, Fairweather D, Senefeld JW, Bruno KA, Klassen SA, Carter RE, Klompas AM, Wiggins CC, Shepherd JR, et al: Early safety indicators of COVID-19 convalescent plasma in 5000 patients. J Clin Invest 130: 4791-4797, 2020.

41. Nguyen FT, van den Akker T, Lally K, Lam H, Lenskaya V, Liu STH, Bouvier NM, Aberg JA, Rodriguez D, Krammer F, et al: Transfusion reactions associated with COVID-19 convalescent plasma therapy for SARS-CoV-2. Transfusion 61: 78-93, 2021.

42. Corral-Gudino L, Bahamonde A, Arnaiz-Revillas F Gómez-Barquero J, Abadía-Otero J, García-Ibarbia C, Mora V, Cerezo-Hernández A, Hernández JL, López-Muñíz G, et al: Methylprednisolone in adults hospitalized with COVID-19 pneumonia: An open-label randomized trial (GLUCOCOVID). Wien Klin Wochenschr 133: 303-311, 2021.

43. Liu J, Zheng X, Huang Y, Shan $\mathrm{H}$ and Huang J: Successful use of methylprednisolone for treating severe COVID-19. J Allergy Clin Immunol 146: 325-327, 2020.

44. Besedovsky L, Lange T and Born J: Sleep and immune function. Pflug Arch Eur J Physiol 463: 121-137, 2012.

45. Almeida $\mathrm{C}$ and Malheiro A: Sleep, immunity and shift workers: A review. Sleep Sci 9: 164-168, 2016.
46. Irwin MR: Why sleep is important for health: A psychoneuroimmunology perspective. Annu Rev Psychol 66: 143-172, 2015.

47. Huang X, Li H, Meyers K, Xia W, Meng Z, Li C, Bai J, He S, Cai W, Huang C, et al: Burden of sleep disturbances and associated risk factors: A cross-sectional survey among HIV-infected persons on antiretroviral therapy across China. Sci Rep 7: 3657, 2017.

48. Taylor DJ, Kelly K, Kohut ML and Song KS: Is insomnia a risk factor for decreased influenza vaccine response? Behav Sleep Med 15: 270-287, 2017.

49. Groeneveld GH, van 't Wout JW, Aarts NJ, van Rooden CJ, Verheij TJM, Cobbaert CM, Kuijper EJ, de Vries JJC and van Dissel JT: Prediction model for pneumonia in primary care patients with an acute respiratory tract infection: Role of symptoms, signs, and biomarkers. BMC Infect Dis 19: 976 , 2019.

50. Guan W, Ni ZY, Hu Y, Ling WH, Qu CQ, He JX, Liu L, Shan H, Lei CL, Hui DSC, et al: Clinical characteristics of 2019 novel coronavirus infection in China. MedRxiv 2: 6, 2020.

51. Wang D, Hu B, Hu C, Zhu F, Liu X, Zhang J, Wang B, Xiang H, Cheng Z, Xiong Y, et al: Clinical characteristics of 138 hospitalized patients with 2019 novel coronavirus-infected pneumonia in Wuhan, China. JAMA 323: 1061-1069, 2020.

52. Chen N, Zhou M, Dong X, Qu J, Gong F, Han Y, Qiu Y, Wang J, Liu Y, Wei Y, et al: Epidemiological and clinical characteristics of 99 cases of 2019 novel coronavirus pneumonia in Wuhan, China: A descriptive study. Lancet 395: 507-513, 2020.

53. Anness A and Siddiqui F: COVID-19 complicated by hepatic dysfunction in a 28-week pregnant woman. BMJ Case Rep 13: e237007, 2020

54. Zandi M, Farahani A, Zakeri A, Akhavan Rezayat S, Mohammadi R, Das U, Dimmock JR, Afzali S, Nakhaei MA, Doroudi A, et al: Clinical symptoms and types of samples are critical factors for the molecular diagnosis of symptomatic COVID-19 patients: A systematic literature review. Int J Microbiol 2021: 5528786, 2021.

55. Malekifar P, Pakzad R, Shahbahrami R, Zandi M, Jafarpour A, Rezayat SA, Akbarpour S, Shabestari AN, Pakzad I, Hesari E, et al: Viral coinfection among COVID-19 patient groups: An update systematic review and meta-analysis. Biomed Res Int 2021: 5313832, 2021.

56. Soltani S, Zakeri A, Zandi M, Kesheh MM, Tabibzadeh A, Dastranj M, Faramarzi S, Didehdar M, Hafezi H, Hosseini P and Farahani A: The role of bacterial and fungal human respiratory microbiota in COVID-19 patients. Biomed Res Int 2021: 6670798,2021

This work is licensed under a Creative Commons Attribution-NonCommercial-NoDerivatives 4.0 International (CC BY-NC-ND 4.0) License. 